\title{
Imaging of Object at Multiple Depths with Single Exposure using Lightfield Concept
}

\author{
Neeraj Singh ${ }^{1}$, Dr. Poonam Juneja ${ }^{2}$ \\ ${ }^{1}$ Ramjas college, Department of Physics, University of Delhi, University Enclave, Delhi-110007, India \\ ${ }^{2}$ Maitreyi college, Department of Physics, University of Delhi, Bapudham Complex Chanakyapuri, New Delhi-110021, India
}

\begin{abstract}
The lightfield camera is a computational camera that achieves imaging of object at multiple depths with single exposure. Instead of recording total intensity at each pixel as in usual camera, the lightfield camera system keeps track of directional distribution of light at each pixel. This information enables us to perform computational refocusing. The commercial product has been recently introduced by Lytro systems. We have studied working related to the computational photography and after this we have developed some code in matalab for recording the intensity pattern at detector and also developed code for image recovery. Finally we did experimental work and recorded the Light field and then applied image recovery algorithm. After this we got the photograph of an object at different depth from the single recoded pattern at a particular location.
\end{abstract}

Keywords: Lightfield, directional distribution, computational, image recovery

\section{Introduction}

Photography has been in existence for more than two centuries. In last couple of decades, the technological developments in electronic array sensors has effectively replaced film based cameras by digital cameras. While film has been replaced by digital sensor arrays (e.g. CCD or CMOS), the Physics of imaging has largely remained the same. The ready availability of numerical data at the sensor plane, however, opens up new possibilities for imaging if a camera is considered as a combination of the optics hardware and numerical algorithms for image recovery. A combination of optics and image recovery methods may show imaging performance that cannot be achieved purely by optical design or purely by image processing. The possibilities for better performance include better resolution, better depth of field, better field of view, hardware improvements such as compact lightweight cameras as those in smartphones, etc. [4] In this work we study and demonstrate one concept in this general area that has received some attention recently - the capability of computational refocusing. Classes of systems with this capability are known as light field imaging systems [1][2]. Currently there are two commercial products that use this idea to achieve single shot imaging followed by computational refocus. Lytro systems [9] have introduced a camera with this feature. The cost of this camera system is approximately the same as any typical Nikon/Canon/.....camera ( $\sim$ Rs. 25 -30k). Another company Raytrix [10] in Germany has also introduced similar products suited to industrial and scientific applications. We point out that our camera system has much lower resolution compared to the commercial lightfield systems. Our aim in this work has been to understand the main ideas involved in the working of this system and to setup a complete working model including in-house developed software for image recovery. The other possible technologies that show improved depth of focus are Cubic phase mask [6] based system and integral imaging technologies [7][3].

Wave front coding with cubic phase masks is a very wellknown technique used to extend the depth of field of incoherent imaging system. It was suggested by the Dowski and cathey [6]. This technique with cubic phase masks works to blur the image uniformly using a cubic shaped wave plate so that the intermediate image, the optical transfer function, is out of focus by constant amount. Digital image processing removes the blur and introduces noise depending upon the physical characteristics of the processor. Dynamic range is sacrificed to extend the depth of field depending upon the type of filter used. It can also correct optical aberration. One of the important aspects of wave front coding technique is the design of appropriate phase mask.

Integral imaging [7] is a passive multi perspective imaging technique, which means it displays a 3D image without the use of special glasses of the part of the viewer. In integral imaging, multiple 2D images of a scene records from different perspectives. It achieves this by placing an array of micro lenses in front of the image, where each lens looks different depending on viewing angle. The color image display by the integral imaging is free from speckle degradation. The integral imaging technique has been extensively used for many applications in research e.g. security, industrial applications, robotics, medicine and defense due to their diverse and significant benefits.

\section{Concept of Lightfield}

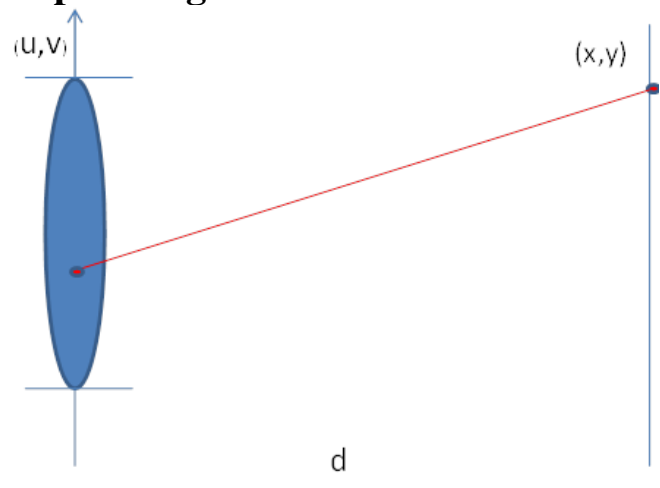

Figure 1: Camera lens and sensor plane 


\section{International Journal of Science and Research (IJSR) \\ ISSN (Online): 2319-7064}

Index Copernicus Value (2013): 6.14 | Impact Factor (2014): 5.611

Light field is an important new concept that is required for understanding computational refocus, as it is achieved in this camera. In an ordinary digital camera, a sensor pixel element integrates all the energy falling on it during the exposure time. The information about the direction of travel of a lightray from the camera aperture to the pixel location is completely lost. The directional distribution of light energy arriving at a pixel location may be described by what is called as a light field function. Figure (1) shows a nominal camera lens and a sensor plane at a distance ' $d$ ' behind the lens aperture. From figure (1), physically the Light field $\mathrm{L}(\mathrm{u}, \mathrm{v} ; \mathrm{x}, \mathrm{y})$ is the distribution of light energy from an elemental area dudv located in the lens aperture plane at coordinates $(\mathrm{u}, \mathrm{v})$ to a point $(\mathrm{x}, \mathrm{y})$ in the sensor plane. Conventional cameras cannot record the Light field. The Light field $\mathrm{L}(\mathrm{u}, \mathrm{v} ; \mathrm{x}, \mathrm{y})$ is the 4D function, the $(\mathrm{u}, \mathrm{v})$ coordinates describe a location in the lens aperture plane and $(\mathrm{x}, \mathrm{y})$ describe a point in the sensor plane. In this work we will use the parameterization of light field shown in figure (1) which describes each ray by its intersection points with two planes: the sensor $(x, y)$ and the aperture $(\mathrm{u}, \mathrm{v})$ inside the lens. Since each ray that contributes to the photographs passes through the lens aperture as well as terminates somewhere in to the sensor plane. So the use of two plane parameterization technique is useful inside the Light field camera. The Light field function can be related to the intensity $\mathrm{I}(\mathrm{x}, \mathrm{y})$ recorded by an ordinary camera as follows:

$$
I(x, y)=\iint d u d v L(u, v ; x, y)
$$

It is noticed that in order to obtain the intensity at location $(\mathrm{x}, \mathrm{y})$ we have integrated the light field function over the whole aperture. It is therefore clear that the knowledge of the Light field function between the lens plane and a sensor plane enables us to determine the intensity pattern that may be observed in the sensor plane.

\section{Recording a photograph's Lightfield}

For recording lightfields instead of regular photographs, the basic idea is to insert an array of micro lenses in front of the photo sensor in a conventional camera. Each micro lens covers multiple photo sensor pixels, and separates the light rays that strike it in to tiny image on the pixels underneath. The distances between the main lens, the microlens array and the sensor plane are such that each microlens images the main lens aperture on the sensor array. The image behind each of the micro lenses represents the distribution of energy arriving at the microlens from the main lens aperture as seen from the microlens location. The collection of all images behind the microlens array thus provides the total 4D Light field function.

\section{Extraction of Lightfield for fixed (u,v)}

Figure (2) shows the distribution of main lens aperture $(\mathrm{u}, \mathrm{v})$ in to the detector plane at different locations. We use an array of detectors capable of forming an image beneath each microlens. Considering that the micro lenses are small in size and located near the optical axis of the main lens, the corresponding pixel under each micro lens does not receives light from the same part of the aperture, it is clear from figure (3) that some shift will occur. In our setup the micro lens will be $150 \mu \mathrm{m}$ in diameter and pixel size is about $5 \mu \mathrm{m}$ so the image under each micro lens is 30x30 in dimension. In order to extract $\mathrm{L}(\mathrm{u}, \mathrm{v} ; \mathrm{x}, \mathrm{y})$, for a fixed $(\mathrm{u}, \mathrm{v})$ we will collect the pixels corresponding to $(\mathrm{u}, \mathrm{v})$ from each micro lens image considering the shift in to account and form an NXN array. This NXN image is a sample of $\mathrm{L}(\mathrm{u}, \mathrm{v} ; \mathrm{x}, \mathrm{y})$ for fixed (u,v). In similar fashion we can extract a series of images corresponding to different $(\mathrm{u}, \mathrm{v})$ locations. This series of image gives another representation for the full Light field function that is obtained by rearranging the sensor data. If this Light field function is integrated over $(\mathrm{u}, \mathrm{v})$ will give $\mathrm{I}(\mathrm{x}, \mathrm{y})$ over the micro lens array plane.
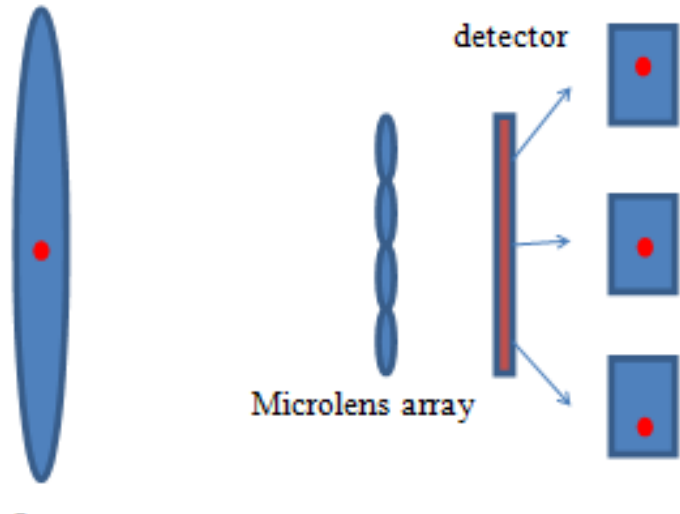

Lens

Figure 2: Imaging of main lens aperture by microlens array

The integration over (u,v) here refers to addition of all images for different values of $(\mathrm{u}, \mathrm{v})$ as obtained above. The $\mathrm{I}(\mathrm{x}, \mathrm{y})$ will have the focused image of the object in a plane at a distance $d_{o b j}$ from the main lens, where $1 / d+1 / d_{o b j}=1 / F$, and $\mathrm{F}$ is the focal length of the main lens.

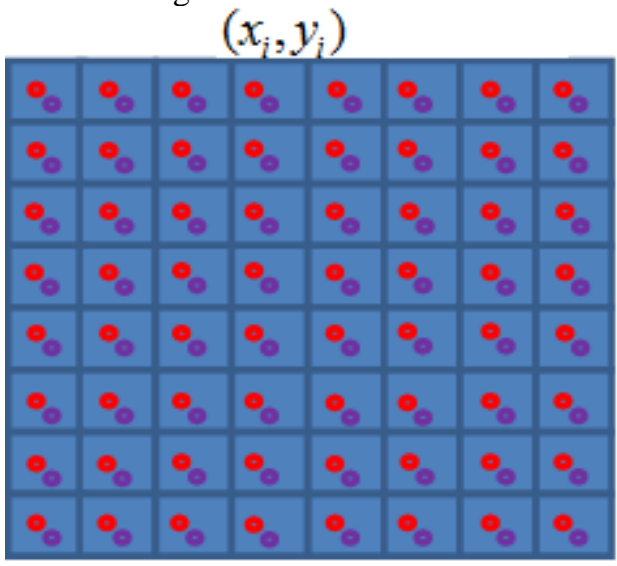

Figure 3: Distribution of main lens aperture

\section{Computational Refocus}




\section{International Journal of Science and Research (IJSR) \\ ISSN (Online): 2319-7064}

Index Copernicus Value (2013): 6.14 | Impact Factor (2014): 5.611

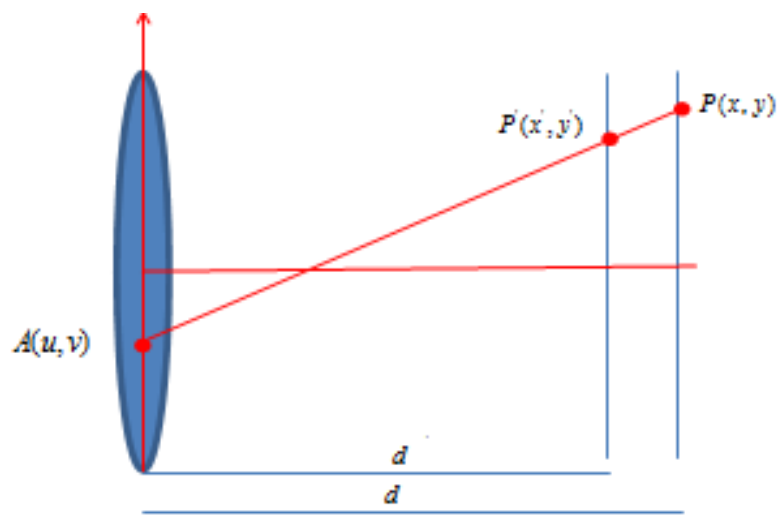

Figure 4: Camera lens and sensor plane

The important idea behind the computational refocus method is that if the light field function $\mathrm{L}(\mathrm{u}, \mathrm{V} ; \mathrm{x}, \mathrm{y})$ between two planes, the main lens aperture plane and another one at a distance $\mathrm{d}$ from the main lens aperture plane, is known ,one can find the new Light field function $\mathrm{L}\left(\mathrm{u}, \mathrm{v} ; \mathrm{x}^{\prime}, \mathrm{y}^{\prime}\right)$ using this data[Ref.1], where (x',y') are coordinate at a different plane at a distance d' from the main lens as shown in figure (4). It is clear now that if this new Light field function is integrated over $(\mathrm{u}, \mathrm{v})$ we will get an image corresponding to different object plane.

From figure (4) we obtain the following geometrical relations.

$$
\begin{aligned}
& \frac{\left(x-x^{\prime}\right)}{\left(d-d^{\prime}\right)}=\frac{(x-u)}{d} \\
& \left(x-x^{\prime}\right)=\frac{(x-u)\left(d-d^{\prime}\right)}{d} \\
& x^{\prime}=x-\frac{(x-u)\left(d-d^{\prime}\right)}{d} \\
& x^{\prime}=x\left[1-\frac{\left(d-d^{\prime}\right)}{d}\right]+u \frac{\left(d-d^{\prime}\right)}{d} \\
& x^{\prime}=\alpha x+\beta u \\
& \text { Where } \\
& \alpha=1-\frac{\left(d-d^{\prime}\right)}{d}, \quad \beta=\frac{\left(d-d^{\prime}\right)}{d} \\
& \text { Similarly } \\
& y^{\prime}=\alpha y+\beta v
\end{aligned}
$$

So we observe that $\mathrm{L}\left(\mathrm{u}, \mathrm{v} ; \mathrm{x}^{\prime}, \mathrm{y}^{\prime}\right)$ is equal to $\mathrm{L}(\mathrm{u}, \mathrm{v}$; $\alpha x+\beta u, \alpha y+\beta v)$. We have already know the grid of coordinates $\left(\mathrm{x}_{\mathrm{j}}, \mathrm{y}_{\mathrm{j}}\right)$ corresponding to micro lens locations, and from this we find $\left(\mathrm{x}_{\mathrm{j}}, \mathrm{y}_{\mathrm{j}}\right)$. The grid $\left(\mathrm{x}_{\mathrm{j}}, \mathrm{y}_{\mathrm{j}}\right)$ is related to the grid $\left(\mathrm{x}_{\mathrm{j}}, \mathrm{y}_{\mathrm{j}}\right)$ by scaling $\alpha$ and shift $\beta \mathrm{u}$ and $\beta \mathrm{v}$ if the aperture coordinates $(\mathrm{u}, \mathrm{v})$ are held fixed. The new light field function $\mathrm{L}\left(\mathrm{u}, \mathrm{v} ; \mathrm{x}^{\prime}, \mathrm{y}^{\prime}\right)$ can thus be obtained by interpolating between the two grids. So corresponding to each $\mathrm{L}(\mathrm{u}, \mathrm{v} ; \mathrm{x}, \mathrm{y})$ for a fixed $(\mathrm{u}, \mathrm{v})$ we get an image L(u,v; x',y'). Now integrating this new L(u,v; x', y') over all $(\mathrm{u}, \mathrm{v})$ we will get a new image I'( $\left.\mathrm{x}^{\prime}, \mathrm{y}^{\prime}\right)$ which corresponding to new object plane.

$$
I^{\prime}\left(x^{\prime}, y^{\prime}\right)=\iint d u d v L\left(u, v ; x^{\prime}, y^{\prime}\right)
$$

The computational refocus thus involves the steps as follows:

a) Record sensor data

b) Rearrange the sensor data for individual value of $(\mathrm{u}, \mathrm{v})$

c) Interpolate between the two grid locations

d) Integrate over all the $(u, v)$ locations

\section{Simulation Results}

For computational refocus we have a recorded sensor pattern as shown in figure (5). From this raw data we extract a series of light field function for different point in the aperture plane. The process of extraction of light field from the recorded sensor pattern has discussed above.

\section{1) Extracted lightfield for fixed (u,v)}

From the raw data as shown in figure (5) the extracted light fields for fixed $(\mathrm{u}, \mathrm{v})[(\mathrm{u}, \mathrm{v})$ is a co-ordinate of the aperture plane] are shown in figure (6). We have divided main lens aperture in to $30 \times 30$. So we have series of 900 such light fields, but we are showing bellow only three [a $(14,15)$, b $(15,14)$, c $(15,15)]$ of them.
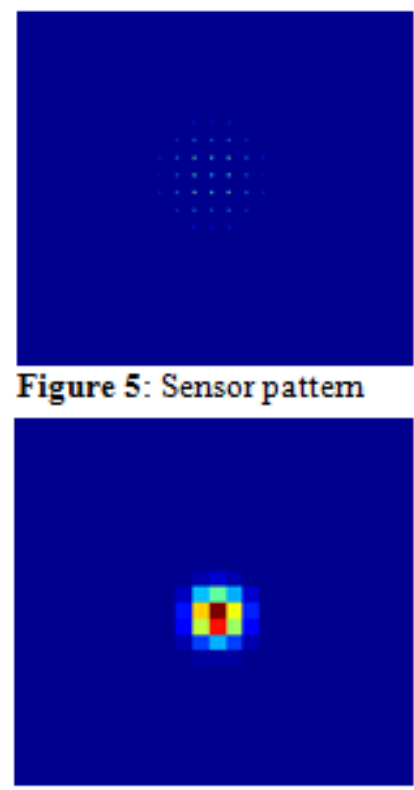

(b): $(15,14)$

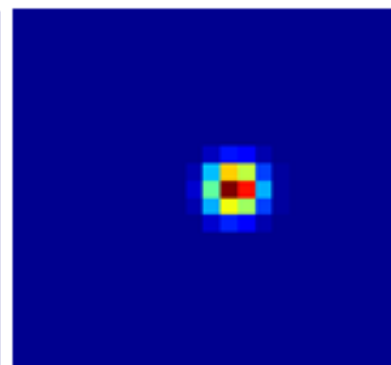

(a): $(14,15)$

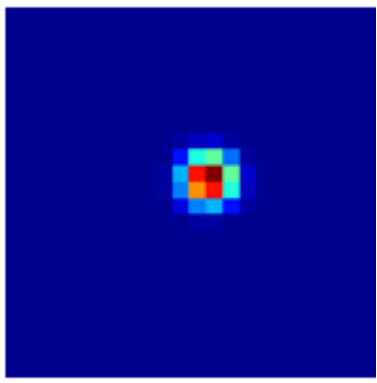

(c): $(15,15)$
Figure 6: Extracted lightfield for fixed (u,v)

\section{2) Computational image from the extracted lightfields}

The idea, for obtaining the computational image from extracted light fields has already discussed. We add 30x30 extracted light fields (as shown above in figure 6) after proper scaling and shifting, and then we get the required image of the object as shown in figure (8). The sensor pattern which is shown in figure (7) is for an object at a distance $15 \mathrm{~mm}$ from the main lens (focal length $10 \mathrm{~mm}$ ). It is clear from the figures (8a) that if the micro lens plane is the imaging plane of the main lens then the computational image will be sharp, otherwise it will be blur as shown in figures (8b and 8c). 


\section{International Journal of Science and Research (IJSR) \\ ISSN (Online): 2319-7064}

Index Copernicus Value (2013): 6.14 | Impact Factor (2014): 5.611

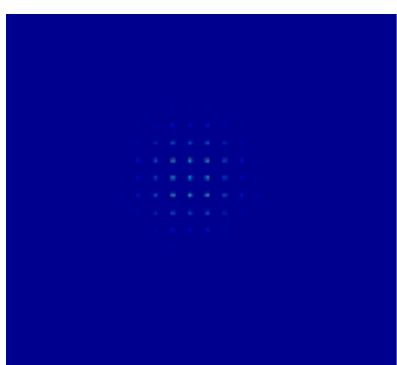

Figure 7: Sensor pattem

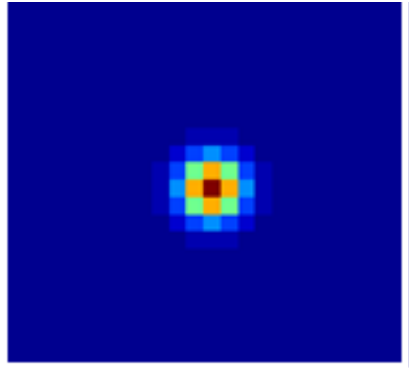

(b): Computed image at $(60 \mathrm{~mm})$

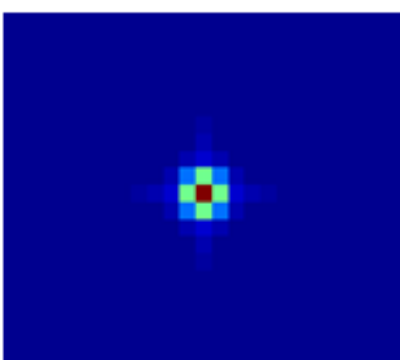

(a): Computed mage at nominal image plane $(30 \mathrm{~mm})$

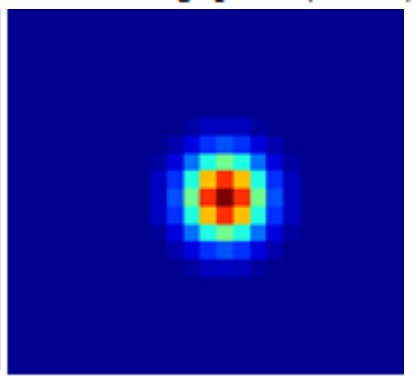

(c): Computed image at $(80 \mathrm{~mm})$

Figure 8: Computed image

\section{Experimental results for a $\mathrm{V}$ shape object}

The recorded Light field for a V shape object is shown in figure (9a). From this recorded light field we obtain the number of images for different-different alphas as shown in figure (9). Each alpha image represents the image of an object at a particular plane.

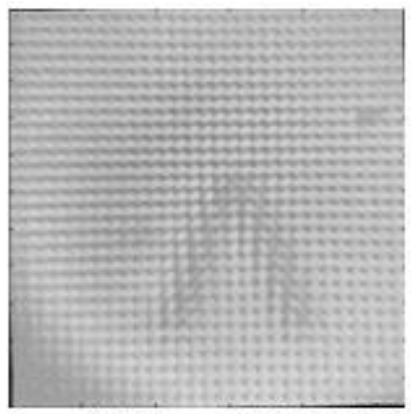

(a): Recorded lightfield

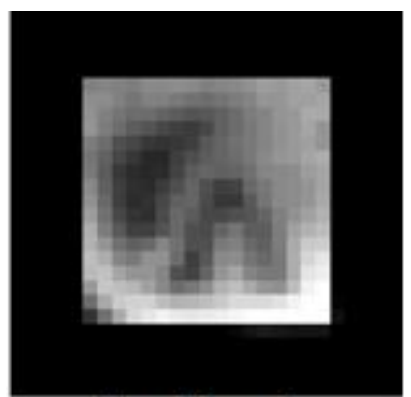

(c): alpha $=.7$

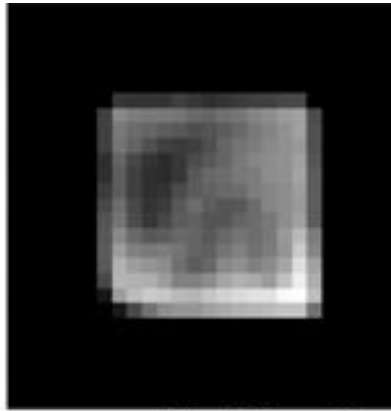

(b): alpha $=.6$

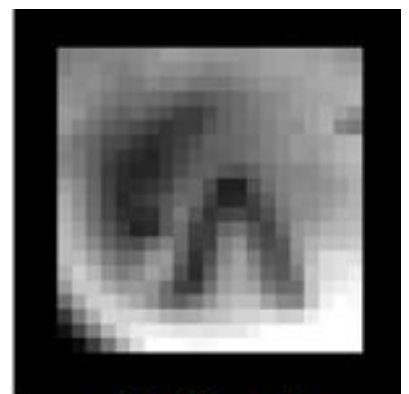

(d): alpha $=.8$

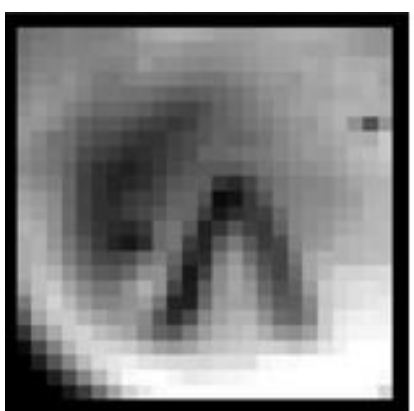

(e): alpha $=.9$

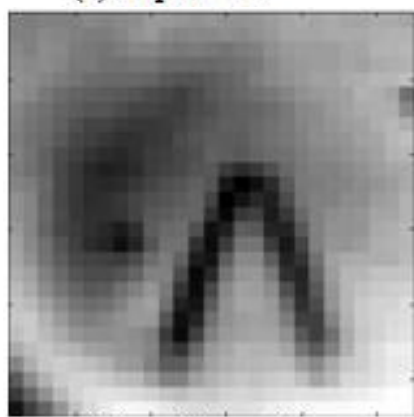

(g): alpha $=1.1$

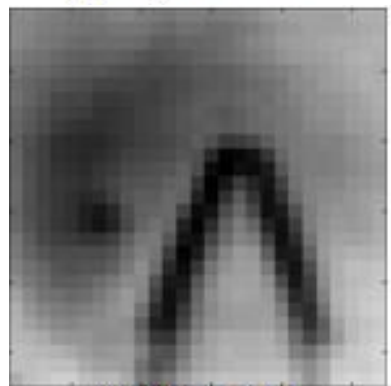

(i): alpha $=1.3$

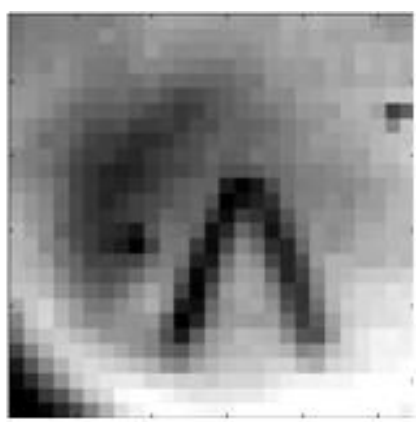

(f): alpha $=1$

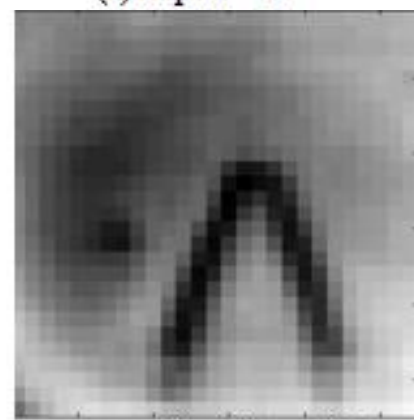

(h): alpha $=1.2$

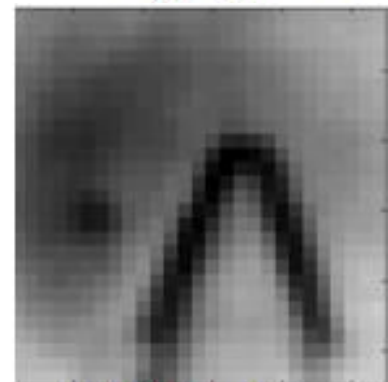

(j): alpha $=1.4$
Figure 9: Computed images for different alphas

\section{Conclusions}

We have set up a low-resolution version of the commercial light field camera and demonstrated the possibility of computational refocus with our experimental data combined with the software developed in-house. The Light field camera demonstrates that a simple low-cost optical modification to existing camera model along with computational algorithm for image recovery can achieve focusing at multiple depths using single image exposure. Image recovery algorithms use the full Light field to unleash new ways to make and view pictures. Relying on numerical algorithm as well as optical components we can improve performance with respect to superior depth imaging/ sectioning capabilities that would not possible with hardware alone or software (image processing) alone. It also creates new opportunities to innovate on camera lenses, controls and design. In particular, the problems associated with optical focus are not fundamental characteristics of photography. The light field imaging concept described in this work is to record the Light field flowing into conventional photographs, and to use the computer to control the final convergence of rays in images. This new kind of photography means unprecedented capabilities after exposure: refocusing, choosing a new depth of field, and possibly correcting lens aberrations. 


\section{References}

[1] Ren Ng, Light Field Photography with a hand-held Plenoptic camera, Stanford Tech Report CTSR 2005-02.

[2] ADELSON, T., AND WANG, J. Y. A. 1992. Single lens stereo with a plenoptic camera.IEEE Transactions on pattern Analysis and Machine Intelligence 14,2(Feb), 99-106.

[3] NAEMURA, T., YOSHIDA, T., AND HARASHIMA, H. 2001. 3-D computer graphics based on integral photography. Optics Express 8, 2, 255-262.

[4] TANIDA, J., SHOGENJI, R. 2003. Color imaging with an integrated compound imaging system. Optics Express 11, 18 (September 8), 2109-2117.

[5] Ren Ng Thesis of Stanford University (July 2006).

[6] Edward R. Dowski, Jr., and W. Thomas cathey.

[7] Applied optics, vol. 52, issue 4, pp.546-560 (2013). Advances in three dimensional integral imaging.

[8] GOODMAN, J. W. 1996. Introduction to Fourier optics, 2nd edition. McGraw-Hill.

[9] Lytro, Inc. (U.S.A).

[10] Raytrix GmbH (German company).

[11] ISAKSEN, A., McMILLAN, L., AND GORTLER, S. J. 2000. Dynamically reparameterized light fields. In SIGGRAPH 2000, 297-306.

[12]LEVOY, M., AND HANRAHAN, P. 1996. Light field rendering. In SIGGRAPH 96, 31-42.

[13] LIPPMANN, G. 1908. La photographie integrale. Comptes-Rendus, Academie des sciences 146, 446-551.

[14] VAISH, V., WILBURN, B., JOSHI, N., AND LEVOY, M. 2004. Using plane+parallax for calibrating dense camera arrays. In proceedings of CVPR.

[15] ADAMS, A. 1995. The camera. Bulfinch press.

[16] LEVOY, M., CHEN, B., VAISH, V., HOROWITZ, M., McDOWALL, I., AND BOLAS, M. 2004. Synthetic aperture confocal imaging. ACM Transactions on Graphics (Proceedings of SIGGRAPH 2004)23, 3, 822831.

[17]Kingslake, R., [A history of the photographic lens], Academic Press (1989).

[18] IVES, H.E. 1930. Parallax panoramagrams made with a large diameter lens. J. Opt. Soc. Amer.20, 332-342.

[19]IVES, H. E. 1931. Optical properties of a Lipmann lenticulated sheet. J. Opt. Soc. Amer. 21, 171-176.

[20] Edmund Y .Lam . Computational photography.

\section{Author Profile}

Neeraj Singh, did M.Sc from Allahabad University in 2011, M.Tech from IIT DELHI in 2013. Now, I am working as Assistant Professor in Ramjas College department of Physics, University of Delhi.

Poonam Juneja, Associate professor, in department of Physics of Maitreyi college, University of Delhi. 